\title{
COMPARISONS OF JOINT KINETICS IN THE LOWER EXTREMITY BETWEEN STAIR ASCENT AND DESCENT
}

\author{
H.-C. Lin * \\ Institute of Biomedical Engineering \\ National Taiwan University \\ Taipei, Taiwan 10617, R.O.C. \\ School of Physical Therapy \\ China Medical University \\ Taichung, Taiwan 40402, R.O.C. \\ T.-W. Lu ${ }^{* *}$ \\ Institute of Biomedical Engineering \\ National Taiwan University \\ Taipei, Taiwan 10617, R.O.C. \\ H.-C. Hsu ${ }^{* * *}$ \\ School of Physical Therapy \\ China Medical University \\ Taichung, Taiwan 40402, R.O.C. \\ Department of Orthopedics \\ China Medical University Hospital \\ Taichung, Taiwan 40402, R.O.C.
}

\begin{abstract}
Stair locomotion has been used in the rehabilitation of the lower extremity as a motor performance test and multi-joint exercise. Controversies exist regarding joint loads during stair locomotion. The purposes of the study were to investigate the three-dimensional kinetics of the lower limb joints during stair locomotion, and to compare them with those during level walking. Ten normal young adults walked, and ascended and descended stairs in a gait laboratory while kinematic and kinetic data were collected and analyzed. The results showed that the intersegmental resultant forces at the joints during the activities were generally similar in pattern but the force magnitudes, moments and angular impulses were significantly different. The general impression that the loads in the lower limb were larger during stair descent than stair ascent only holds for certain kinetic variables. Most of the peak joint moments and angular impulses over the entire stance phase were bigger during stair ascent than descent. The study provides a complete knowledge of the three-dimensional loading patterns at and dynamic functions of the lower limb joints during level walking and stair locomotion. It will be helpful for the planning and evaluation of treatment programs for patients with neuromusculoskeletal pathologies in the lower extremities.
\end{abstract}

Keywords : Stair locomotion; Joint loads; Level walking; Kinetic analysis.

\section{INTRODUCTION}

Stair locomotion, being a common functional activity of daily living, has been used in the rehabilitation of the lower extremity as a motor performance test [1] and multi-joint close kinetic chain $(\mathrm{CKC})$ exercise to increase muscle strength and facilitate weight-bearing of the joints $[2,3]$. Several studies have shown that stair locomotion places higher loads on the joints of the lower limb than level walking does, with larger ranges of motion [4 6], joint moments [6 8] and muscle efforts [9]. It is generally assumed that stair descent loads the joints of the lower limb much more than stair ascent. In many clinical situations, patients have been advised to avoid stair descent during the course of rehabilitation. Therefore, step-ups are used in clinics for training [10]. As a result, most of the previous studies have focused on the biomechanics of stair ascent only (e.g. $[8,9,11])$. Since both stair ascent and descent are frequent activities of daily living, direct quantitative evidence is needed to clarify whether or not stair descent loads the lower limb joints more than stair ascent. To the best knowledge of the authors, no study exists in the literature that provides a complete comparison of the three-dimensional loadings on all the lower limb joints between stair ascent and descent.

\footnotetext{
*Ph.D. candidate $\quad$ ** Associate Professor, corresponding author *** Associate Professor, Medical Doctor 
Most previous kinematic studies on stair activities in normal subjects were limited to two-dimensional (2D) movement patterns $[4,5,7,12]$. With simultaneous measurement of the ground reaction forces using force platforms, kinetics of the joints can be deduced using the so-called inverse dynamics analysis, which provides more information for the evaluation and investigation of this activity. Early kinetic studies were mostly limited to 2D analyses [12]. Andriacchi et al. [4] calculated the three-dimensional net external moments by taking the cross product of the vector defining the position of the joint center and the vector defining the foot-ground reaction force, neglecting contributions from inertial terms and segment weights. However, it has been suggested that their method increases errors in the calculated moments at joints proximal to the ankle $[13,14]$. Other studies have reported moments in the frontal as well as sagittal planes $[8,15,16]$, but moment components in the transverse plane are not reported in the literature. There are a limited number of studies on complete three-dimensional kinetic analyses on one or two isolated joints in the lower limb during stair locomotion [6,11]. However, since stair locomotion is predominately a multi-joint coordinated movement, knowledge of the mechanics of this activity requires a complete and detailed analysis of the mechanical interactions between the joints.

There is no study in the literature that compares the 3D joint resultant forces, moments and impulses between stair ascent and descent. The only study that compared the moments and powers of the lower extremity joints between stair ascent and descent was limited to the sagittal plane components [7]. Although previous studies helped in establishing a general picture of the movement patterns during stair locomotion, detailed information on the 3D kinetic differences between stair ascent and descent are necessary and important for clinical applications. The purposes of the present study were thus to perform a complete $3 \mathrm{D}$ analysis on the kinetics of the joints of the lower limb during stair ascent and descent, and to compare the mechanical interactions of the joints between these two activities and level walking.

\section{MATERIALS AND METHODS}

Ten healthy adults ( 5 males and 5 females; age: 21.9 \pm 1.6 years; height: $167.9 \pm 8.3 \mathrm{~cm}$; mass: $61.5 \pm 9.8 \mathrm{~kg}$ ) participated in this study with informed consent. They had no history of low back or lower limb problems that would affect their functional activities.

The experiment was performed in a gait laboratory equipped with a seven-camera motion analysis system and two force platforms. A marker system with 26 retro-reflective markers was developed, with at least 3 non-collinear markers per segment, to describe the motion of the lower limb segments. These markers were attached to specific bony landmarks on each limb, including the anterior superior iliac spines (ASIS), the posterior superior iliac spines (PSIS), the greater trochanter, the mid-thigh, the medial and lateral femoral epicondyles, the tibial tuberosity, the head of fibula, the medial and lateral malleoli, the calcaneus, the navicular tuberosity, and the base of the fifth metatarsal. Three-dimensional coordinates of these markers were collected for each subject during a static standing trial for the definition of the body-embedded coordinate systems of the segments. The markers on the medial femoral epicondyle and medial malleolus were then removed during the dynamic trials to avoid interference with the movements.

The seven-camera motion analysis system (VICON 370, Oxford Metrics, U.K.) was used to measure the trajectories of the markers with a sampling rate of $60 \mathrm{~Hz}$. The ground reaction forces (GRF) were measured at a sampling rate of $240 \mathrm{~Hz}$ with two force platforms (AMTI, Mass., U.S.A.), which were located in the middle of a ten-meter walkway during level walking trials. During stair locomotion, the force platforms served as the second step of the three-step stair (Fig. 1). Each subject was asked to walk at a self-selected pace and then to climb the custom-made three-step stair (height: $18 \mathrm{~cm}$; depth: $46 \mathrm{~cm}$ ) first ascending and then descending with both legs being tested (Fig. 1). The starting position of the subject was adjusted by the examiner so that the tested foot could place naturally on the force platform during the tested activities. The motion data from at least six trials for each activity were collected.

The body-embedded orthogonal coordinate system for each segment was defined using the markers on that segment, with the positive $x$-axis directed anteriorly,

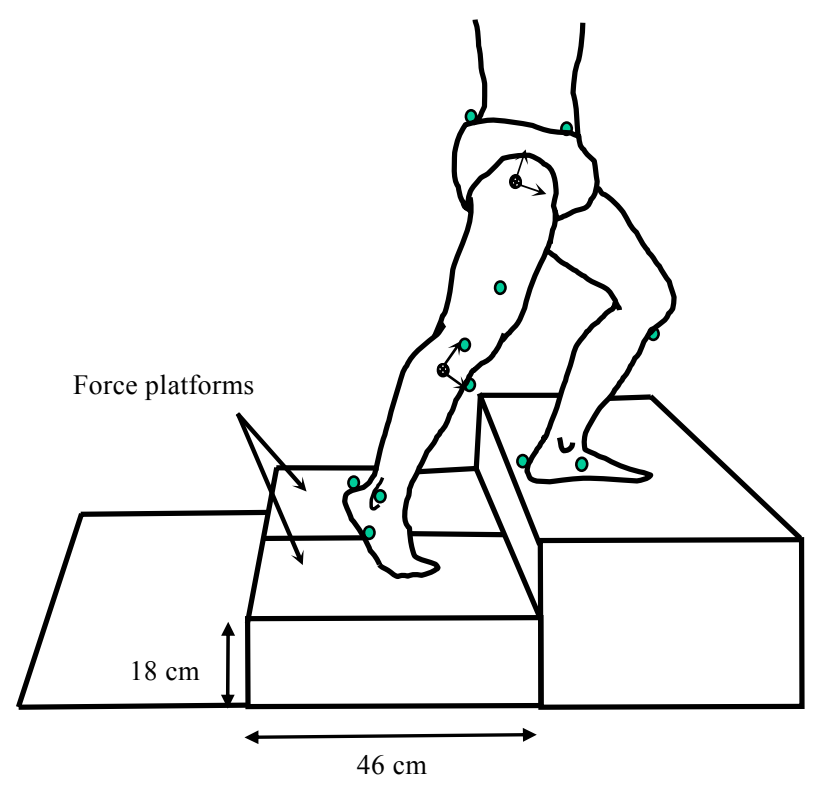

Fig. 1 Schematic diagram showing a subject ascending a three-step stair with the tested foot stepped on the second step fitted with two force platforms. Part of markers placed on the bony landmarks for the tracking of the limb segments is also shown 
$y$-axis superiorly and $z$-axis to the right. The rotational movements of the joints were described with the Cardanic $z-y-x$ rotation sequence, following suggestions by Grood and Suntay [17]. The corresponding clinical terms for the calculated Cardanic angles were assigned following the recommendations from the Standards and Terminology Committee of the International Society of Biomechanics [18]. The intersegmental resultant forces, moments and angular impulses were calculated using the inverse dynamics technique [19] with a 3-D lower limb model from the measured kinematic and kinetic data. Inertial properties of each segment were determined using Dempster's coefficients [20].

The intersegmental resultant forces and moments at the ankle were obtained by solving Newton-Euler equations for the free body of the foot. Similarly, those for the knee were calculated considering equilibrium of the shank segment and for the hip considering the thigh segment. Peak values of the intersegmental resultant forces and moments for each joint during stance phase were extracted for subsequent statistical analysis. The angular impulses, a measure of the total muscular effort over the entire stance phase, for each net joint moment component at each joint were also calculated as follows.

$$
I_{i}^{j}=\int_{T} M_{i}^{j}(t) d t, \quad i=x, y, z
$$

where $I_{i}^{j}$ and $M_{i}^{j}(t)$ are the i-th component of the angular impulse and moment at joint $j$ respectively and $T$ is the total time of stance phase.

To account for anatomical variations between the subjects, the calculated intersegmental resultant forces were normalized to body weight (Newtons), and joint moments and impulses to body weight (BW in Newtons) and leg length (LL in meters). The curves of the intersegmental resultant force and moment components were normalized by the total time of stance phase (SP) so that the stance phase begins at $0 \% \mathrm{SP}$ and ends at $100 \%$ SP. The curves of each force or moment component at each joint from all the subjects were then ensemble-averaged to obtain the mean and standard deviation for the description of the joint kinetics. The peak values of each intersegmental resultant force and moment components, as well as the impulses, were used for subsequent comparisons. These dependent variables were analyzed using repeated measures analysis of variance (ANOVA) to distinguish the main effects from activity types. Post hoc analysis was then performed for multiple comparisons when a significant difference in a dependent variable was detected by ANOVA. A paired t-test was also performed to compare the difference between stair ascent and descent. The significant level was set to 0.05 .

\section{RESULTS}

\subsection{Intersegmental Resultant Forces}

Not only loading patterns but also the peak forces were different between the tested activities (Fig. 2, Table 1). In the anterior/posterior (A-P) direction during level walking the hip joint transmitted mostly posterior forces during early stance phase (0-50\% SP) and anterior forces during late stance phase (51-100\% SP). However, posterior forces at the hip were required throughout most of the stance phases during stair activities. The peak of the posterior force at the hip during the stance phase of stair ascent was significantly larger than those during level walking and stair descent (Table 1). During late stance phase, a peak posterior force of $24.2 \% \mathrm{BW}$ was noted at the hip during stair descent while almost no A-P forces were required during stair ascent (Figs. 2a). Anterior forces were transmitted at the knee and ankle joints throughout most of the stance phase except at the initial contact of level walking (Table 1, Figs. $2 \mathrm{~b} \sim 2 \mathrm{c}$ ). The two peaks of the anterior forces at the knee during level walking were significantly smaller than those of both stair activities except that no significant difference was found between the second peaks of level walking and stair ascent, both of which were significantly smaller than those during stair descent (Table 1, Fig. 2b). Anterior forces at the ankle during these activities also had two-peak patterns with statistically similar peak values, the only exception being the first peak during stair descent that was significantly bigger than the other two (Table 1, Fig. 2c).

For the proximal/distal (P-D) loadings, resultant distal forces were required throughout most of the stance phase in all the tested activities owing to body weight-bearing. For all three joints, the first peak distal forces during stair descent were all significantly bigger than those in the other two activities, while the second ones were all significantly smaller (Table 1). The first peaks during stair ascent were smaller than those during level walking while the second ones were bigger (Table 1, Figs. 2d 2f).

The magnitudes of the medial/lateral (M-L) force components were much smaller than the other two components. Generally, the hip joint transmitted medial forces while the knee and ankle joints lateral ones. Very similar loading patterns in the M-L direction were found for all joints during all the activities. However, it is noted that the first peaks at the knee and ankle during stair ascent were significantly smaller than those during level walking and stair descent. The second peaks at the ankle during stair descent were significantly smaller than those during the other two activities (Table 1, Figs. $2 \mathrm{~g} \sim 2 \mathrm{i}$ ).

\subsection{Joint Moments}

The curves of the sagittal moment components of the three joints were quite different between the tested activities (Figs. 3a 3c). During level walking, extensor moments were required at the hip during $0 \sim 50 \% \mathrm{SP}$ and then changed to flexor moments during the rest of the SP (51 100\% SP) while during stair ascent extensor moments were required throughout most of the SP. Stair descent had a totally different pattern of hip 
(a) Hip ant/post

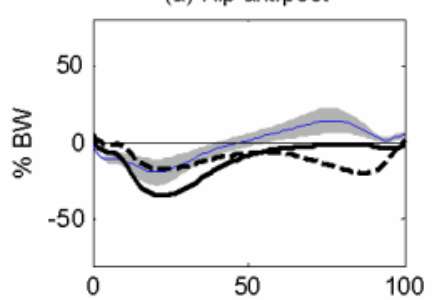

(b) Knee ant/post

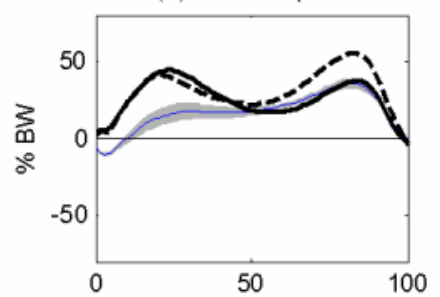

(c) Ankle ant/post

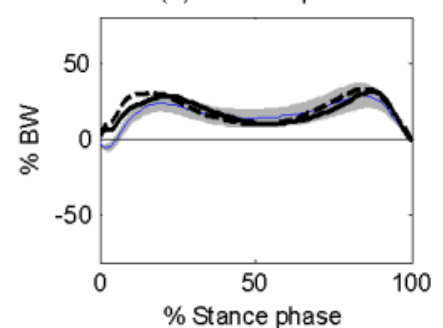

(d) Hip prox/dist

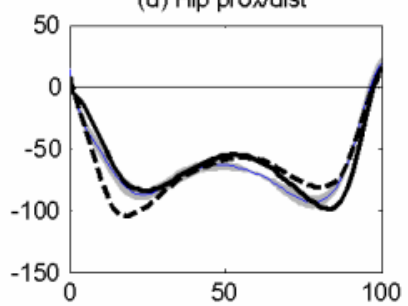

(e) Knee prox/dist
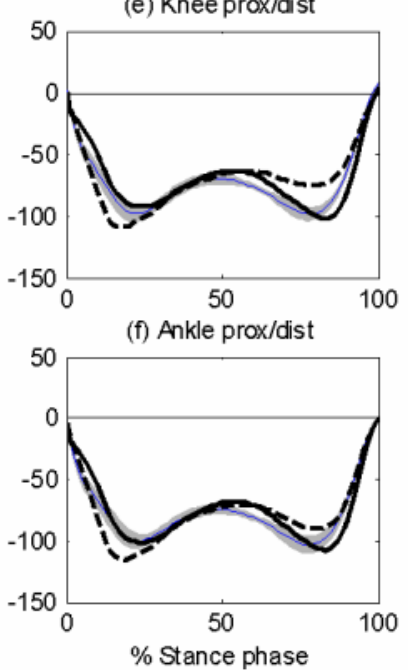

(g) Hip lat/med

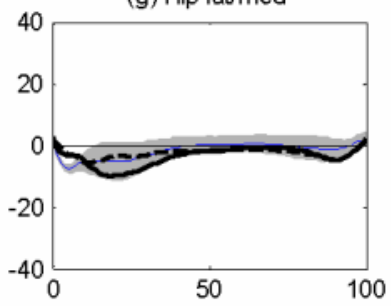

(h) Knee lat/med
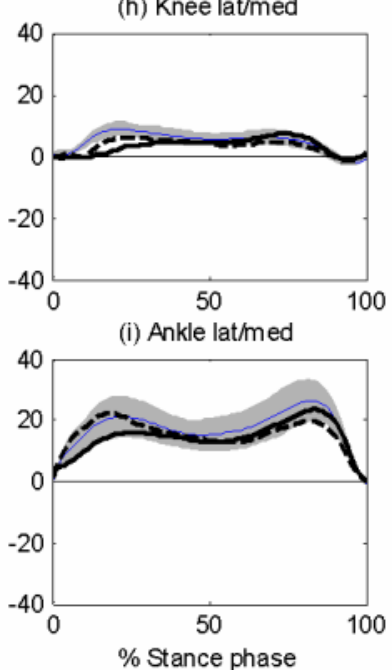

Fig. 2 Ensemble-averaged intersegmental resultant forces in the sagittal $(\mathrm{a} \sim \mathrm{c})$, frontal $(\mathrm{d} \sim \mathrm{f})$ and transverse planes $(\mathrm{g} \sim \mathrm{h})$ during stance phase of level walking (solid thin curves), stair ascent (solid thick curves) and descent (dashed thick curves). Shadow areas are one standard deviation from the mean curves for level walking

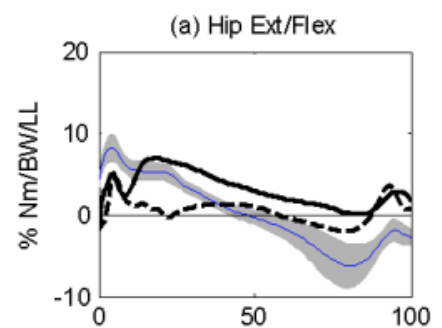

(b) Knee Ext/Flex

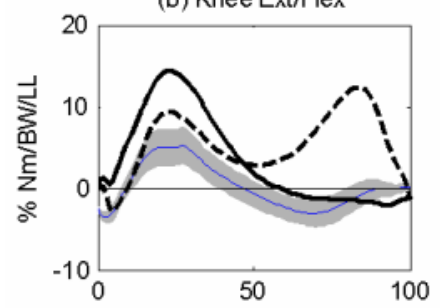

(c) Ankle Plant/Dorsi

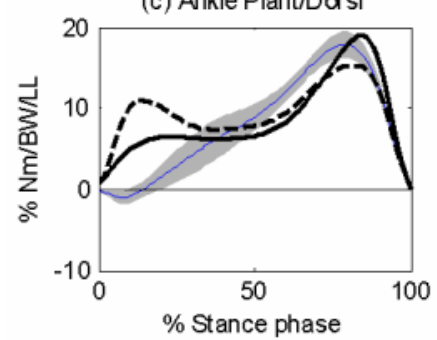

(d) Hip Abd/Add

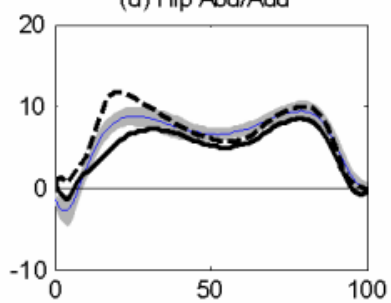

(e) Knee Abd/Add

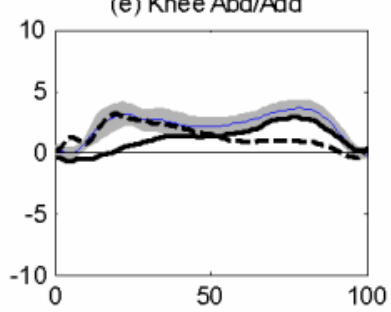

(f) Ankle Ever/lnv

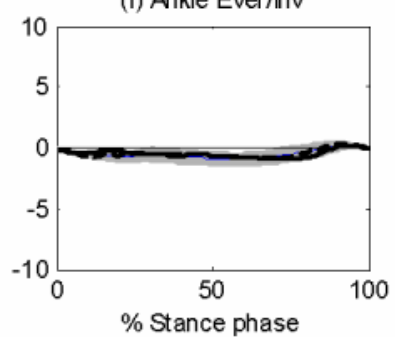

(g) Hip IR/ER

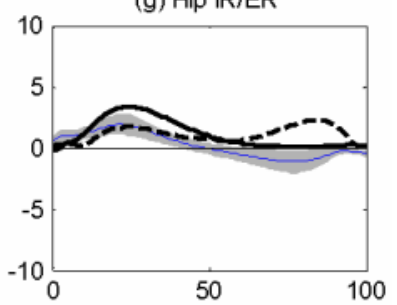

(h) Knee IR/ER

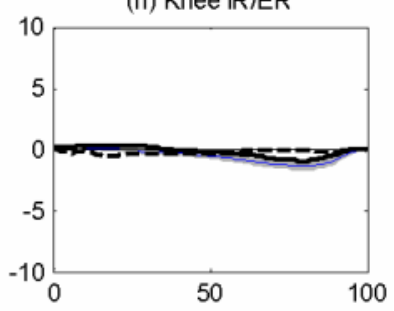

(i) Ankle IR/ER

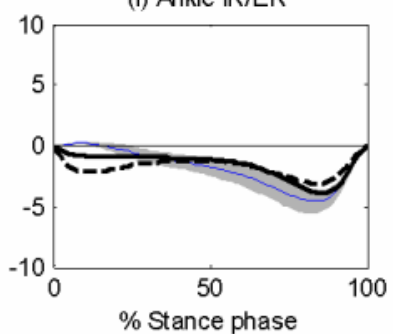

Fig. 3 Ensemble-averaged joint moments in the sagittal $(\mathrm{a} \sim \mathrm{c})$, frontal $(\mathrm{d} \sim \mathrm{f})$ and transverse planes $(\mathrm{g} \sim \mathrm{h})$ during stance phase of level walking (solid thin curves), stair ascent (solid thick curves) and descent (dashed thick curves). Shadow areas are one standard deviation from the mean curves for level walking 
Table 1 Peak values of the intersegmental resultant forces $(\% \mathrm{BW})$ during level walking and stair locomotion

\begin{tabular}{|c|c|c|c|c|c|c|c|c|c|c|c|c|c|}
\hline \multirow[b]{3}{*}{ Joint } & \multirow[b]{3}{*}{ Direction } & \multicolumn{4}{|c|}{ Level walking } & \multicolumn{4}{|c|}{ Stair ascent } & \multicolumn{4}{|c|}{ Stair descent } \\
\hline & & \multicolumn{2}{|c|}{ Early SP } & \multicolumn{2}{|c|}{ Late SP } & \multicolumn{2}{|c|}{ Early SP } & \multicolumn{2}{|c|}{ Late SP } & \multicolumn{2}{|c|}{ Early SP } & \multicolumn{2}{|c|}{ Late SP } \\
\hline & & Mean & Std & Mean & Std & Mean & Std & Mean & Std & Mean & Std & Mean & Std \\
\hline \multirow[t]{6}{*}{ Hip } & anterior & $3.76^{\dagger}$ & 3.22 & $17.06^{\dagger}$ & 7.54 & $-0.98^{\mathrm{a}}$ & 4.27 & $5.15^{\mathrm{a}}$ & 8.36 & $4.56^{* \mathrm{c}}$ & 3.91 & $2.25^{\mathrm{b}}$ & 1.24 \\
\hline & posterior & $20.39^{\dagger}$ & 9.52 & $1.04^{\dagger}$ & 2.05 & $41.38^{\mathrm{a}}$ & 8.46 & $10.27^{\mathrm{a}}$ & 4.03 & $16.76^{* c}$ & 9.23 & $24.21 * b c$ & 4.75 \\
\hline & proximal & $13.99^{\dagger}$ & 3.81 & $19.02^{\dagger}$ & 4.66 & $-2.22^{\mathrm{a}}$ & 4.96 & 15.46 & 0.58 & $1.15^{\mathrm{b}}$ & 3.15 & 15.38 & 0.52 \\
\hline & distal & $88.94^{\dagger}$ & 5.53 & $95.90^{\dagger}$ & 7.19 & $83.61^{\mathrm{a}}$ & 5.38 & 105.94 & 5.76 & $122.87 * \mathrm{bc}$ & 28.40 & $84.89^{*}$ & 8.57 \\
\hline & lateral & $3.39^{\dagger}$ & 1.49 & 5.05 & 3.49 & $0.81^{\mathrm{a}}$ & 2.05 & 2.91 & 0.85 & $3.29 * \mathrm{c}$ & 2.61 & $3.96^{*}$ & 0.97 \\
\hline & medial & 10.47 & 4.47 & 4.81 & 3.05 & 13.20 & 8.11 & 6.72 & 4.72 & 8.45 & 6.92 & 6.69 & 3.92 \\
\hline \multirow[t]{6}{*}{ Knee } & anterior & $17.80^{\dagger}$ & 5.55 & $34.36^{\dagger}$ & 3.94 & $45.18^{\mathrm{a}}$ & 3.91 & 33.13 & 7.02 & $42.02^{\mathrm{b}}$ & 14.72 & $59.40 * \mathrm{bc}$ & 9.96 \\
\hline & posterior & $11.30^{\dagger}$ & 2.06 & $-0.19^{\dagger}$ & 0.60 & $-2.82^{\mathrm{a}}$ & 3.81 & $2.84^{\mathrm{a}}$ & 0.46 & $-2.67^{b}$ & 2.87 & $5.40 * \mathrm{bc}$ & 0.23 \\
\hline & proximal & $2.90^{\dagger}$ & 2.19 & $8.04^{\dagger}$ & 1.55 & $-11.73^{\mathrm{a}}$ & 5.41 & $5.31^{\mathrm{a}}$ & 0.19 & $-7.94^{b}$ & 3.42 & $2.19 * \mathrm{bc}$ & 0.40 \\
\hline & distal & $100.18^{\dagger}$ & 7.40 & $101.29^{\dagger}$ & 6.34 & $92.99^{\mathrm{a}}$ & 6.61 & $111.19^{a}$ & 5.69 & $127.93 * \mathrm{bc}$ & 26.73 & $78.89 * b c$ & 5.18 \\
\hline & lateral & $10.82^{\dagger}$ & 3.22 & 8.83 & 3.34 & $7.73^{a c}$ & 5.00 & 9.16 & 5.09 & $11.11^{*}$ & 3.98 & 8.52 & 5.99 \\
\hline & medial & -0.02 & 1.15 & 2.47 & 0.94 & 3.38 & 3.22 & 2.77 & 2.14 & 2.30 & 3.65 & 3.70 & 2.68 \\
\hline \multirow[t]{6}{*}{ Ankle } & anterior & $24.89^{\dagger}$ & 7.95 & 28.81 & 10.21 & 25.94 & 11.52 & 31.48 & 10.72 & $34.11 * b c$ & 13.27 & 34.08 & 11.89 \\
\hline & posterior & $6.72^{\dagger}$ & 3.19 & $-0.41^{\dagger}$ & 0.61 & $-2.11^{\mathrm{a}}$ & 3.54 & $1.14^{\mathrm{a}}$ & 0.32 & $-4.99^{b}$ & 5.83 & $1.46^{* b c}$ & 0.26 \\
\hline & proximal & $-2.92^{\dagger}$ & 2.28 & $1.81^{\dagger}$ & 0.53 & $-16.44^{\mathrm{a}}$ & 6.01 & $1.00^{\mathrm{a}}$ & 0.11 & $-9.93 * b \mathrm{c}$ & 4.02 & $0.63 * b c$ & 0.19 \\
\hline & distal & $101.86^{\dagger}$ & 7.91 & $105.38^{\dagger}$ & 8.94 & 103.36 & 7.39 & $114.63^{\mathrm{a}}$ & 8.08 & $133.03 * \mathrm{bc}$ & 27.42 & $94.82 * \mathrm{bc}$ & 10.94 \\
\hline & lateral & $21.55^{\dagger}$ & 7.81 & $26.20^{\dagger}$ & 8.00 & $16.39^{\mathrm{ac}}$ & 5.43 & 24.76 & 7.68 & $25.15^{*}$ & 7.35 & $20.11 * b \mathrm{c}$ & 7.26 \\
\hline & medial & $-0.41^{\dagger}$ & 0.91 & 1.00 & 0.40 & -1.66 & 1.97 & 0.47 & 0.46 & $-3.01 * b \mathrm{c}$ & 2.06 & 0.79 & 1.23 \\
\hline
\end{tabular}

* $p<0.05$ with paired t-test between stair ascent and descent

$\dagger p<0.05$ with main effect in repeated measures ANOVA comparing level walking with stair ascent and descent

a $p<0.05$ with post hoc of repeated measures ANOVA comparing level walking and stair ascent

b $p<0.05$ with post hoc of repeated measures ANOVA comparing level walking and stair descent

c $p<0.05$ with post hoc of repeated measures ANOVA comparing and stair ascent and descent

loading. There were two peak extensor moments, one immediately after heel-strike and the other before toe-off, the former being much smaller than the corresponding value during level walking and stair ascent. The hip was almost free from subjecting to sagittal moments during the rest of the SP (Table 2, Fig. 3a). For knee extensor/flexor moments, similar single-peak curves were found for stair ascent and level walking, with the peak of the stair ascent being almost 2 to 3 times bigger than that of level walking. For stair descent, a peak knee extensor moment up to 13.7 (\%BW $\times$ LL) was required during late SP, a major difference from the other two activities (Table 2, Fig. 3b). It is noted that peak knee extensor moments in both stair activities were significantly larger than during level walking. The ankle sagittal moment curves for the stair activities displayed similar two-peak patterns, one during early stance and the other during late stance, while there was only a peak during late stance for level walking. The peak ankle plantar flexor moments during late stance were 19.6, 16.4 and $18.3(\% \mathrm{BW} \times \mathrm{LL})$ for stair ascent, stair descent and level walking, respectively. The peak ankle plantar flexor moments for stair descent were significantly smaller than those for stair ascent and level walking. However, significantly larger peak ankle plantar flexor moment during early stance was required for stair descent (Table 2, Fig. 3c). 
Table 2 Peak values of the joint moments $(\% \mathrm{BW} \times \mathrm{LL})$ during level walking and stair locomotion

\begin{tabular}{|c|c|c|c|c|c|c|c|c|c|c|c|c|c|}
\hline & & \multicolumn{4}{|c|}{ Level walking } & \multicolumn{4}{|c|}{ Stair ascent } & \multicolumn{4}{|c|}{ Stair descent } \\
\hline & & \multicolumn{2}{|c|}{ Early SP } & \multicolumn{2}{|c|}{ Late SP } & \multicolumn{2}{|c|}{ Early SP } & \multicolumn{2}{|c|}{ Late SP } & \multicolumn{2}{|c|}{ Early SP } & \multicolumn{2}{|c|}{ Late SP } \\
\hline & & Mean & Std & Mean & Std & Mean & Std & Mean & Std & Mean & Std & Mean & Std \\
\hline \multirow[t]{6}{*}{ Hip } & abductor & $9.55^{\dagger}$ & 1.40 & 9.98 & 1.54 & $8.11^{\mathrm{a}}$ & 2.41 & 9.33 & 2.63 & $14.04 * \mathrm{bc}$ & 2.83 & $11.20^{*}$ & 2.56 \\
\hline & adductor & $3.21^{\dagger}$ & 2.33 & $0.22^{\dagger}$ & 0.84 & 2.09 & 1.49 & $1.18^{\mathrm{ac}}$ & 0.85 & $-0.19^{*} \mathrm{bc}$ & 1.23 & $0.53 *$ & 0.54 \\
\hline & internal rotator & $2.16^{\dagger}$ & 0.81 & $0.12^{\dagger}$ & 0.37 & $4.27^{\mathrm{ac}}$ & 1.12 & $1.19^{\mathrm{a}}$ & 0.62 & $2.04 *$ & 0.93 & $2.71 * \mathrm{bc}$ & 1.02 \\
\hline & external rotator & 0.26 & 0.43 & $1.50^{\dagger}$ & 0.84 & 0.16 & 0.47 & $0.47^{\mathrm{a}}$ & 0.81 & 0.25 & 0.56 & $0.23^{\mathrm{b}}$ & 0.27 \\
\hline & extensor & $9.35^{\dagger}$ & 2.34 & $0.22^{\dagger}$ & 1.20 & 9.77 & 2.34 & $4.20^{\mathrm{a}}$ & 1.40 & $5.32 * \mathrm{bc}$ & 1.90 & $4.29^{\mathrm{b}}$ & 1.98 \\
\hline & flexor & $0.56^{\dagger}$ & 0.71 & $7.28^{\dagger}$ & 2.08 & $-1.77^{\mathrm{a}}$ & 2.60 & $0.71^{\mathrm{a}}$ & 1.48 & $2.55^{* b c}$ & 2.27 & $3.42 * b \mathrm{c}$ & 1.27 \\
\hline \multirow[t]{6}{*}{ Knee } & abductor & $3.76^{\dagger}$ & 1.27 & $4.20^{\dagger}$ & 1.04 & 2.49 & 1.67 & 3.80 & 1.27 & $4.23 *^{c}$ & 1.63 & $2.75^{b}$ & 1.58 \\
\hline & adductor & $0.37^{\dagger}$ & 0.53 & $0.35^{\dagger}$ & 0.32 & $2.19^{\mathrm{ac}}$ & 1.56 & 0.27 & 0.47 & $0.51 *$ & 0.88 & $1.12 * \mathrm{bc}$ & 0.81 \\
\hline & internal rotator & $0.29^{\dagger}$ & 0.18 & $0.06^{\dagger}$ & 0.09 & $0.79^{\mathrm{ac}}$ & 0.41 & $0.23^{\mathrm{a}}$ & 0.15 & $0.27^{\mathrm{c}}$ & 0.32 & $0.57^{\mathrm{b}}$ & 0.54 \\
\hline & external rotator & $0.66^{\dagger}$ & 0.27 & $1.56^{\dagger}$ & 0.50 & $0.26^{\mathrm{ac}}$ & 0.14 & 1.28 & 0.54 & $0.90^{*}$ & 0.48 & $0.81^{\mathrm{b}}$ & 0.57 \\
\hline & extensor & $5.47^{\dagger}$ & 2.85 & $0.70^{\dagger}$ & 0.68 & $17.23^{\mathrm{a}}$ & 2.65 & $3.01^{\mathrm{a}}$ & 2.29 & $10.48^{* \mathrm{bc}}$ & 2.70 & $13.74^{\mathrm{bc}}$ & 2.97 \\
\hline & flexor & $4.11^{\dagger}$ & 1.19 & $4.36^{\dagger}$ & 2.06 & $0.21^{\mathrm{ac}}$ & 1.29 & 4.98 & 3.04 & $3.28 *$ & 1.29 & $1.63 * b c$ & 0.25 \\
\hline \multirow[t]{6}{*}{ Ankle } & evertor & 0.01 & 0.32 & $0.41^{\dagger}$ & 0.32 & -0.09 & 0.26 & 0.65 & 0.49 & 0.75 & 1.51 & $0.78^{\mathrm{b}}$ & 0.37 \\
\hline & invertor & $1.32^{\dagger}$ & 0.59 & 1.46 & 0.59 & 1.26 & 0.52 & 1.45 & 0.78 & $1.91 *^{\mathrm{c}}$ & 0.93 & 1.37 & 0.94 \\
\hline & internal rotator & $0.44^{\dagger}$ & 0.14 & 0.04 & 0.09 & $0.02^{\mathrm{a}}$ & 0.16 & 0.04 & 0.07 & $-0.25^{* b c}$ & 0.37 & 0.09 & 0.15 \\
\hline & external rotator & $1.48^{\dagger}$ & 0.87 & $4.41^{\dagger}$ & 1.16 & $1.07^{\mathrm{a}}$ & 0.50 & $4.09^{\mathrm{a}}$ & 1.11 & $2.61 * b \mathrm{c}$ & 1.09 & $3.20 * \mathrm{bc}$ & 1.20 \\
\hline & plantarflexor & $8.21^{\dagger}$ & 2.07 & $18.31^{\dagger}$ & 1.98 & $6.55^{\mathrm{a}}$ & 1.16 & 19.58 & 1.67 & $12.77 * \mathrm{bc}$ & 3.85 & $16.41 * \mathrm{bc}$ & 2.19 \\
\hline & dorsiflexor & $1.28^{\dagger}$ & 0.93 & $-0.13^{\dagger}$ & 0.15 & $-0.81^{\mathrm{a}}$ & 0.83 & $0.02^{\mathrm{a}}$ & 0.01 & $-0.74^{\mathrm{b}}$ & 1.48 & $-0.01 * \mathrm{bc}$ & 0.01 \\
\hline
\end{tabular}

* $p<0.05$ with paired t-test between stair ascent and descent

$\dagger p<0.05$ with main effect in repeated measures ANOVA comparing level walking with stair ascent and descent

a $p<0.05$ with post hoc of repeated measures ANOVA comparing level walking and stair ascent

b $p<0.05$ with post hoc of repeated measures ANOVA comparing level walking and stair descent

c $p<0.05$ with post hoc of repeated measures ANOVA comparing and stair ascent and descent

In the frontal plane, abductor moments were required at the hip and knee joints throughout most of the SP. Relatively small ankle evertor/invertor moments were found in all the tested activities (Table 2, Fig. 3f). The two peaks of the hip abductor moments in stair descent were the largest (Table 2, Fig. 3d). There were two peaks in the knee abductor moment curve during level walking. Values of these peak moments were not significantly different from the peak for stair descent in early SP and from that for stair ascent in late SP (Table 2, Fig. 3e).

In the transverse plane, internal rotator (IR) moments during early SP and external rotator (ER) moments during late SP occurred at the hip during level walking.
During stair activities, IR moments occurred at the hip throughout the SP. The first peak of the IR moments at the hip during stair descent was significantly smaller than those during level walking and stair ascent while the second peak was significantly larger (Table 2, Fig. $3 \mathrm{~g})$. ER moments occurred at the knee and ankle joints throughout most of the SP for all the tested activities. At the knee, the ER moment curves for level walking and stair ascent were similar with similar peak values $(1.56 \% \mathrm{BW} \times \mathrm{LL}$ for level walking and $1.28 \% \mathrm{BW} \times \mathrm{LL}$ for stair ascent; Table 2, Fig. $3 \mathrm{~h})$. The peak ER moments at the ankle occurred at about the same time during late stance for all the tested activities, the largest being during level walking and the smallest during stair descent with stair ascent in between. It is 
noted that additional single peaks immediately after heel-strike were found for both stair activities, with that for stair descent being significantly larger (Table 2, Fig. $3 i)$.

\subsection{Joint Impulses}

Angular impulses at all the lower limb joints during level walking were significantly different from those during the stair activities, except for the ankle internal rotator impulses. During stair activities most angular impulses were significantly larger than during level walking (Table 3). Angular impulses with nearly the same magnitude were generated around the ankle joint during stair ascent and descent. Angular impulses at the hip and knee joints were found to be larger in magnitude during stair ascent than during stair descent but those for the hip flexors and hip abductors were bigger during stair descent (Table 3 ).

\section{DISCUSSION}

The present study provides a complete comparison of the three-dimensional forces and moments of the lower limb joints during level walking and stair locomotion. The patterns of the intersegmental forces at the hip and knee joints during stair ascent were in agreement with Costigan et al. [11]. The curves of the sagittal joint moments were similar in pattern with previous studies $[6 \sim 8,11,12,15,16,21]$. The calculated moments in the frontal plane were also in agreement with Kowalk et al. [15] and Yu et al. [16] for the knee and Nadeau et al. [8] for all three joints. Unfortunately, no literature, is available for simultaneous comparisons of the threedimensional forces and moments at the three joints. Although the stair locomotion in the present study was limited to three steps, the obtained joint loading patterns were not affected by this limitation and can still be taken as general patterns for normal daily stair movements, as the number of steps does not affect the reproducibility of the calculated joint moments according to Yu et al. [21]

\subsection{Level Walking vs. Stair Locomotion}

Comparisons of the joint kinetics between level and stair walking revealed similarities in the patterns of the intersegmental forces but significant differences in the intersegmental moments. Humans walk steadily in bipedal gait with the body weight shifting cyclically between both legs. The stance phase of each leg can be divided into three sub-phases, namely initial double stance (or weight acceptance), single support and terminal double stance (or weight release) [22]. The two-peak patterns for most of the force components were present owing to the mechanical requirements in response to weight-acceptance and for propulsion of the body around the beginning of weight-release to maintain progression. Similar phases, and thus force patterns, could also be found in stair locomotion [23].
Two-peak compressive (distally directed) forces were required at the three joints due to body weight-bearing and vertical acceleration of the body (Fig. 2). Since the center of body mass (COM) lies in the middle between the two hip joints, the ground reaction forces (GRF) tended to pass medially to the hip joint centers and, with the contributions from the gravitational and inertial forces, thus resulted in medially-directed forces at the hip and laterally-directed forces at the knee and ankle joints in all activities (Fig. 2). A major difference in the force patterns between the tested activities occurred in the A-P force component at the hip. The A-P force curve for stair descent was two-peaked while that for stair ascent was single-peaked with the peak occurring in early stance. During level walking, the A-P forces at the hip exhibited an alternating pattern, directing posteriorly in early SP and anteriorly in late SP. This reflects the decelerating effects from the GRF during early SP and accelerating effects during late SP. During stair ascent, similar force requirements were noted during early SP but no significant A-P force was needed as the GRF were mainly pushing the COM upward instead of forward as they did during level walking. During stair descent, only posteriorlydirected forces were required at the hip, suggesting that the A-P components of the net forces of the GRF, gravitational forces and inertial forces were anteriorlydirected. This indicates that the posteriorly-directed forces at the hip were so controlled that for safety purposes the body was not accelerating excessively. This could be achieved by keeping the body behind the supporting limb, as observed in all the subjects.

Since the patterns of the GRF and intersegmental forces were mostly similar for the tested activities, the observed differences in the patterns of the joint moment components mainly resulted from the significant differences in the joint kinematics and body postures in relation to the GRF during the activities. As mentioned above, the GRF tended to pass medially to the hip joint centers so significant abductor moments at hip and knee joints were required throughout most of the stance phase. Relatively small invertor moments were required at the ankle mainly because the GRF passed close to the centre of the ankle joint. For moment components in the sagittal plane during level walking, extensor moments at the hip and knee were required during early SP and flexor moments during late SP. For stair locomotion mainly extensor moments at the hip and knee were required. At the ankle, the first peak of the sagittal moment components were dorsiflexor moments during level walking as the GRF passed briefly posterior to the ankle joint center immediately after heel-strike. For stair locomotion, the GRF were placed anterior to the ankle joint centre throughout the SP, so plantar flexor moments were found. The differences in the moment components in the sagittal plane between the three activities were mainly from the differences in the way the body was moved forward and upward with different joint arcs specific to each activity. The results of this study showed 
Table 3 Angular impulses (\% BW) during level walking and stair locomotion

\begin{tabular}{|c|c|c|c|c|c|c|c|}
\hline & \multicolumn{2}{|c|}{ Level walking } & \multicolumn{2}{|c|}{ Stair ascent } & \multicolumn{2}{|c|}{ Stair descent } \\
\hline & & Mean & Std & Mean & Std & Mean & Std \\
\hline \multirow[t]{6}{*}{ Hip } & extensor & $0.403^{\dagger}$ & 0.034 & $1.906^{\mathrm{a}}$ & 0.110 & $0.354 * \mathrm{~b}$ & 0.069 \\
\hline & flexor & $0.490^{\dagger}$ & 0.031 & $0.074^{\mathrm{a}}$ & 0.027 & $0.328 * \mathrm{~b}$ & 0.044 \\
\hline & abductor & $1.374^{\dagger}$ & 0.120 & $2.525^{\mathrm{a}}$ & 0.282 & $2.692 * b$ & 0.212 \\
\hline & adductor & $0.047^{\dagger}$ & 0.010 & $0.074^{\mathrm{a}}$ & 0.021 & $0.023 * b$ & 0.010 \\
\hline & internal rotator & $0.099^{\dagger}$ & 0.024 & $0.605^{\mathrm{a}}$ & 0.081 & $0.346 * b$ & 0.078 \\
\hline & external rotator & $0.115^{\dagger}$ & 0.028 & 0.116 & 0.077 & $0.034 * \mathrm{~b}$ & 0.019 \\
\hline \multirow[t]{6}{*}{ Knee } & extensor & $0.243^{\dagger}$ & 0.052 & $2.311^{\mathrm{a}}$ & 0.211 & $2.384^{b}$ & 0.337 \\
\hline & flexor & $0.297^{\dagger}$ & 0.054 & $0.644^{\mathrm{a}}$ & 0.227 & $0.098 * \mathrm{~b}$ & 0.015 \\
\hline & abductor & $0.533^{\dagger}$ & 0.102 & $0.776^{\mathrm{a}}$ & 0.111 & $0.566^{*}$ & 0.145 \\
\hline & adductor & $0.011^{\dagger}$ & 0.002 & $0.178^{\mathrm{a}}$ & 0.042 & $0.137^{b}$ & 0.077 \\
\hline & internal rotator & $0.006^{\dagger}$ & 0.002 & $0.110^{\mathrm{a}}$ & 0.036 & $0.089 * \mathrm{~b}$ & 0.056 \\
\hline & external rotator & $0.126^{\dagger}$ & 0.021 & $0.169^{\mathrm{a}}$ & 0.022 & $0.135^{*}$ & 0.041 \\
\hline \multirow[t]{6}{*}{ Ankle } & plantar flexor & $1.834^{\dagger}$ & 0.100 & $3.763^{\mathrm{a}}$ & 0.275 & $3.764^{b}$ & 0.465 \\
\hline & dorsiflexor & $0.009^{\dagger}$ & 0.005 & $0.001^{\mathrm{a}}$ & 0.001 & $0.003^{b}$ & 0.011 \\
\hline & evertor & $0.028^{\dagger}$ & 0.010 & $0.053^{\mathrm{a}}$ & 0.026 & $0.071 * \mathrm{~b}$ & 0.029 \\
\hline & invertor & $0.163^{\dagger}$ & 0.043 & $0.461^{\mathrm{a}}$ & 0.171 & $0.454^{b}$ & 0.219 \\
\hline & internal rotator & 0.008 & 0.003 & 0.009 & 0.004 & 0.008 & 0.003 \\
\hline & external rotator & $0.340^{\dagger}$ & 0.038 & $0.634^{\mathrm{a}}$ & 0.090 & $0.604^{b}$ & 0.091 \\
\hline
\end{tabular}

* $p<0.05$ with paired t-test between stair ascent and descent

$\dagger p<0.05$ with main effect in repeated measures ANOVA comparing level walking with stair ascent and descent

a $p<0.05$ with post hoc of repeated measures ANOVA comparing level walking and stair ascent

b $\quad p<0.05$ with post hoc of repeated measures ANOVA comparing level walking and stair descent

that higher joint moments and angular impulses and thus higher muscle efforts were required during stair locomotion, in agreement with Riener et al. [7].

Livingston et al. [5] investigated the influence of stair dimension on temporal variables and sagittal joint kinematics and suggested that individuals accommodated these environmental changes mainly by knee flexion. In the present study, the differences of joint moment components between level walking and stair locomotion were most prominent at the knee (Fig. 3b). This may support their suggestion that the knee was the main joint that was used to accommodate changes of walkway levels, such as going from level walking to stair locomotion.

\subsection{Stair Ascent vs. Descent}

It was generally presumed that the loads in the lower limb are larger during stair descent than during stair ascent. The results in this study showed that most of the peak force components during early SP were indeed larger during stair descent than ascent. However, the opposite was found for the peak force components during late SP (Table 1). The only exception was the peak A-P force components at the hip.

The moments at a joint are the result of the contractions of the muscles across the joint to maintain a posture, to initiate a movement or to control a movement. In other words, joint moments represent the muscular efforts required for the execution of a specific joint function. The joint moments for each lower limb joint during the stair activities were very diverse, suggesting that quite different functions, and thus muscle efforts for each joint, were required. Compared to stair ascent, a larger plantarflexor moment at the ankle and smaller extensor moments at the hip and knee joints were found during early SP of stair descent. This was because larger flexion angles at the hip and knee were required during stair ascent [7,23], increasing the lever arms available to the GRF and thus the external flexing moments, so more muscle efforts were required to counteract. During stair descent, initial contact occurred at the forefoot so the GRF were further anterior to the ankle joint center so bigger plantarflexor moments were necessary compared to stair ascent $[7,23]$. 
The angular impulses represented the total efforts from the muscles across the entire period of the stance phase. Compared to stair descent, significantly larger angular impulses were required at the hip and knee joints for stair ascent, suggesting that the total muscular effort at these joints over the stance phase of stair ascent was bigger, but the opposite was found for the hip flexors and abductors. The former is because virtually no hip flexor angular impulses were required during stair ascent (Fig. 3a) and the latter was because the hip abductor moments during stair descent were bigger throughout the entire stance phase (Fig. 3d).

\section{CONCLUSIONS}

Stair locomotion has been used in the rehabilitation of the lower extremity as a motor performance test and multi-joint $\mathrm{CKC}$ exercise to increase muscle strength and facilitate weight-bearing of the joints. It is generally assumed that stair descent loads the joints of the lower limb much more than stair ascent. This and other incomplete knowledge about stair locomotion in relation to level walking have formed the basis for the design and implementation of several clinical practices. The present study clarified some of the controversies by providing complete three-dimensional joint loading conditions and functions at the lower limb joints during level walking and stair locomotion. Compared to level walking, larger ranges of flexion at the hip and knee and the different way the body was moved forward and upward/downward during stair locomotion led to greater effort of the lower limb muscles, and thus increased joint loadings.

From the comparisons of the joint kinetics in the present study, it seems that the general impression that the loads in the lower limb were larger during stair descent than during stair ascent only holds for certain kinetic variables, such as the first peaks of the P-D forces and hip abductor and ankle plantarflexor moments. From the point of view of muscular effort, in terms of peak joint moments and angular impulses over the entire stance phase, most of the values of these variables were larger during stair ascent than during descent.

The present study has established a more complete general picture of the three-dimensional patterns of joint dynamics during stair locomotion. It will be helpful for the planning and evaluation of treatment programs for patients with neuromusculoskeletal pathologies in the lower extremities.

\section{ACKNOWLEDGEMENT}

The authors gratefully acknowledge the financial support from the National Health Research Institute, the National Science Council and China Medical University.

\section{REFERENCES}

1. Pfeifer, K. and Banzer, W., "Motor performance in different dynamic tests in knee rehabilitation," Scandinavian Journal of Medicine \& Science in Sports, Vol. 9, pp. 19-27, (1999).

2. Goodwin, P. C., Morrissey, M. C., Omar, R. Z., Brown, M., Southall, K., and McAuliffe, T. B., "Effectiveness of supervised physical therapy in the early period after arthroscopic partial meniscectomy," Physical Therapy, Vol. 83, pp. 520-35, (2003).

3. Risberg, M. A., Holm, I., Tjomsland, O., Ljunggren, E., and Ekeland, A., "Prospective study of changes in impairments and disabilities after anterior cruciate ligament reconstruction," Journal of Orthopaedic \& Sports Physical Therapy, Vol. 29, pp. 400-12, (1999).

4. Andriacchi, T. P., Andersson, G. B. J., Fermier, R. W., Stern, D., and Galante, J. O., "A study of lower-limb mechanics during stair-climbing," $J$. Bone \& Joint Surg., Vol. 62A, pp. 749-759 (1980).

5. Livingston, L. A., Stevenson, J. M., and Olney, S. J., "Stairclimbing kinematics on stairs of differing dimensions," Arch. Phys. Med. Rehabil., Vol. 72, pp. 398-402 (1991).

6. Kaufman, K. R., Hughes, C., Morrey, B. F., Morrey, M., and An, K.-N., "Gait characteristics of patients with knee osteoarthritis," J. Biomechanics, Vol. 34, pp. 907-915 (2001).

7. Riener, R., Rabuffetti, M., and Frigo, C., "Stair ascent and descent at different inclinations," Gait \& Posture, Vol. 15, pp. 32-44 (2002).

8. Nadeau, S., McFadyen, B. J., and Malouin, F., "Frontal and sagittal analyses of the stair climbing task in healthy adults aged over 40 years: what are the challenges compared to level walking?" Clin. Biomech., Vol. 18, pp. 950-959 (2003).

9. Moffet, H., Richards, C. L., Malouin, F. and Bravo, G., "Load-carrying during stair ascent: a demanding functional test," Gait \& Posture, Vol. 1, pp. 35-44 (1993).

10. Low, J. and Reed A., Basic Biomechanics Explained, Butterworth-Heinemann, Oxford, UK, pp. 173-174 (1996).

11. Costigan, P. A., Deluzio, K. J., and Wyss, U. P., "Knee and hip kinetics during normal stair climbing," Gait \& Posture, Vol. 16, pp. 31-37 (2002).

12. McFadyen, B. J. and Winter, D. A., "An integrated biomechanical analysis of normal stair ascent and descent," J. Biomechanics, Vol. 21, pp. 733-744 (1988).

13. Winter, D. A., Biomechanics and Motor Control of Human Movement, 2nd Ed., John Wiley \& Sons, Inc, New York, pp. 92-93 (1990). 
14. Wells, R., "The projection of the ground reaction force as a predictor of internal joint moments," Bull. Prosthetics Res., Vol. 18, pp. 15-19 (1981).

15. Kowalk, D. L., Duncan, J. A., and Vaughan, C. L., "Abduction-adduction moments at the knee during stair ascent and descent," J. Biomechanics, Vol. 29, pp. 383-388 (1996).

16. Yu, B., Stuart, M. J., Kienbacher, T., Growney, E. S., and An, K.-N., "Valgus-varus motion of the knee in normal level walking and stair climbing," Clin. Biomech., Vol. 12, pp. 286-293 (1997).

17. Grood, E. S. and Suntay, W. J., "A joint coordinate system for the clinical description of threedimensional motions: application to the knee," Trans. ASME J. Biomech. Eng., Vol. 105, pp. 136-144 (1983).

18. Wu, G., Siegler, S., Allard, P., Kirtley, C., Leardini, A., Rosenbaum, D., et al., "ISB recommendation on definitions of joint coordinate system of various joints for the reporting of human joint motion--part I: ankle, hip, and spine," Journal of Biomechanics, Vol. 35, pp. 543-8, (2002).

19. Craik, R. L. and Oatis, C. A., Gait Analysis Theory and Application, Mosby-Year Book, Inc., pp. 223-238 (1995).

20. Winter, D. A., Biomechanics and Motor Control of Human Movement, 2nd Ed., John Wiley \& Sons, Inc, New York, pp. 51-66 (1990).

21. Yu, B., Kienbacher, T., Growney, E. S., Johnson, M. E., and An, K.-N., "Reproducibility of the kinematics and kinetics of the lower extremity during normal stair-climbing." J. Ortho. Res., Vol. 15, pp. 348-352, (1997).

22. Perry, J. and Schoneberger B., Gait Analysis: Normal and Pathological Function, Delmar Thomson Learning, New York, pp. 9-16 (1992)

23. Lin, H. C., Lu, T. W., and Hsu, H. C., "Threedimensional analysis of kinematic and kinetic coordination of the lower limb joints during stair ascent and descent," Biomedical EngineeringApplications, Basis and Communications, Vol. 16, pp. 101-108 (2004).

(Manuscript received June 25, 2004, Accepted for publication December 24, 2004.) 